\title{
Introduction to Brain and Medical Images
}

\author{
Moo K. Chung \\ University of Wisconsin-Madison \\ mkchung@wisc.edu
}

March 11, 2021

This article is based on the first chapter of book Chung (2013), where brain and medical images are introduced. The most widely used brain imaging modalities are magnetic resonance images (MRI), functional-MRI (fMRI) and diffusion tensor images (DTI). A brief introduction to each imaging modality is explained. Further, we explain what kind of curve, volume and surface data that can be extracted from each modality.

\section{Introduction}

MRI depends on the response of magnetic fields in generating digital images that provide structural information about brain noninvasively. Compared to the computed tomography (CT), MRI has been mainly used for in vivo imaging of the brain due to higher image contrast in soft tissues. Unlike CT, MRI does not use $\mathrm{X}$-ray for imaging so there is no risk of radiation exposure. MRI produces images based on the spin-lattice relaxation time (T1), the spin-spin relaxation time (T2) and the proton density ( $\rho$ ) (Bernstein et al., 2005). The widely used T1- and T2-weighted imaging weight the contribution of one component and minimize the effect of the other two. The T1-weighted MRI is more often used in anatomical studies compared to the T2-weighted MRI. Structural images you are seeing are most likely T1-weighted MRI.

DTI also uses MRI scanners but it specifically enables the measurement of the diffusion of water molecules in the brain tissues. At each voxel in DTI, a rate of diffusion and a preferred direction of diffusion are encoded as $3 \times 3$ symmetric and positive definite matrix called diffusion tensor. In each voxel, there are countless number of neural fibers. So the direction of diffusion follows neural fiber tracts in an average sense within each voxel. By tracing the diffusion direction via tractography, it is possible to represent the white matter fiber tracts visually. fMRI also 
uses MRI scanners in measuring brain activity by detecting changes in blood flow using the blood-oxygen-level-dependent (BOLD) contrast. For last two decades, fMRI has been dominating brain mapping research since it is easy to scan and there is no exposure to radiation. Compared to MRI, fMRI is more frequently contaminated by various types of noise. In order to obtain the underlying signal, various advanced statistical procedures are used. The resulting statistical maps are usually color coded to show the strength of activation in the whole brain.

In this article, various brain image data types obtained from different imaging modalities will be introduced with simple MATLAB functions for image processing and visualization. The most difficult part for statisticians and new researchers trying to analyze brain image data is getting the data into a computer programming environment. We also briefly go over manipulating volume and surface data. The MATLAB code and sample data used in this chapter can be downloaded from http://brainimaging.waisman.wisc.edu/ chung/BIA.

\section{Image Volume Data}

The brain MRI is a $3 \mathrm{D}$ array $\mathcal{I}(\mathbf{x})$ containing tissue intensity values at voxel position $\mathbf{x} \in \mathbb{R}^{3}$ Figure 1 shows a usual example of axial cross-section of MRI. We usually do not analyze tissue intensity values directly in quantifying MRI. MRIs go through various image processing steps such as intensity normalization and tissue segmentation before any quantification is attempted. Image intensity normalization is required to minimize the intensity variations within the same tissue that are caused by magnetic field distortion.

Each voxel can be classified into three different tissue types: cerebrospinal fluid (CSF), grey matter and white matter mainly based on image intensity values. A neural network classifier (Kollakian, 1996) or a Gaussian mixture model (Ashburner and Friston, 2000) have been used for the classification. Figure 1 shows the result obtained by a neural network classifier (Kollakian, 1996). The collection of identically classified voxels has been mainly used in voxel-based morphometry (VBM), which will be discussed in detail in a later chapter. VBM as implemented in the statistical parametric mapping (SPM) package (www. fil.ion.ucl.ac . $\mathrm{uk} / \mathrm{spm}$ ), is a fully automated image analysis technique allowing identification of regional differences in gray matter (GM) and white matter (WM) between populations without a prior region of interest. VBM starts with normalizing each structural MRI to the standard SPM template and segmenting it into white and gray matter and cerebrospinal fluid (CSF) based on a Gaussian mixture model (Ashburner et al., 1997; Ashburner and Friston, 2000). Based on a prior probability of each voxel being the specific tissue type, an iterative Bayesian approach is used 

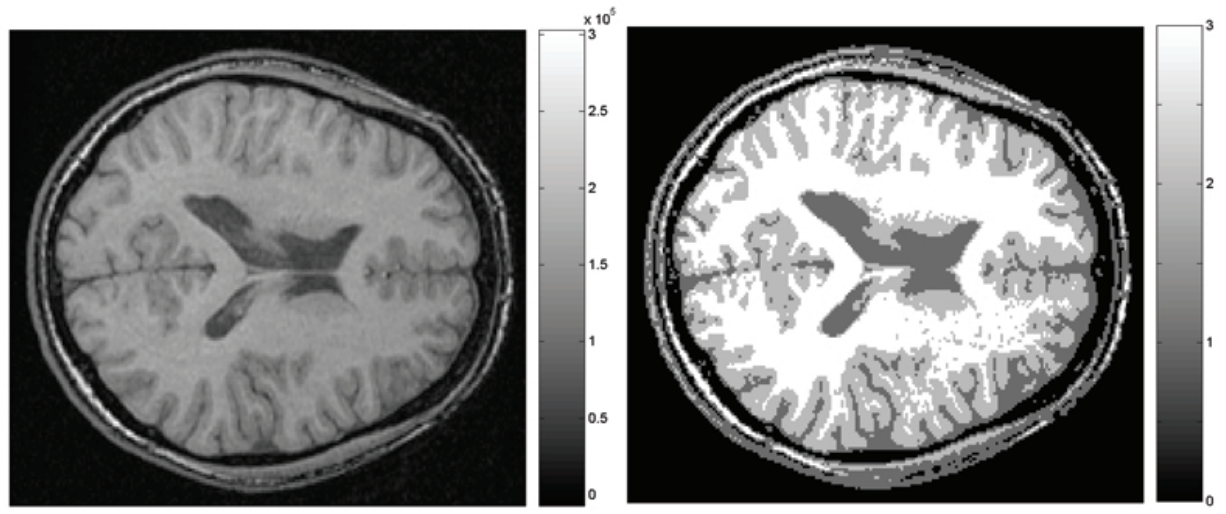

Figure 1: Left; the axial cross-section of MRI. Right: Tissue segmentation into three different classes (white and gray matter and CSF) using the neural network classifier (Kollakian, 1996).

to get an improved estimate of the posterior probability. This probability is usually referred to as the gray or white matter density. Afterwards the density maps are warped into a normalized space and compared across subjects. VBM has been applied in cross-sectional studies on various anatomical studies: normal development (Good et al., 2001; Paus et al., 1999), autism (Chung et al., 2004), depression (Pizzagalli et al., 2004), epilepsy (McMillan et al., 2004) and mild cognitive impairment (MCI) (Johnson et al., 2004).

The most widely used 3D MRI volume image format has been the Analyze 7.5 file format developed by the Biomedical Imaging Resource at Mayo Clinic for Analyze package (www.mayo.edu/bir/Software/Analyze/Analyze. $h t m l)$. An Analyze format consists of two files: an image binary file (*.img) and a header file $(* . h d r)$ that contains information about the binary image file. Various brain image analysis software and MATLAB can read the Analyze 7.5 file format.

Figure 2 shows an example of MRI and the manual segmentation of the left amygdala, which is colored in black. This manual segmentation is stored as a binary image consisting of 0 's and 1's. In MATLAB, to read the header file, we invoke the command

>> header=analyze75info('left_amygdala.hdr') ;

header $=$ 


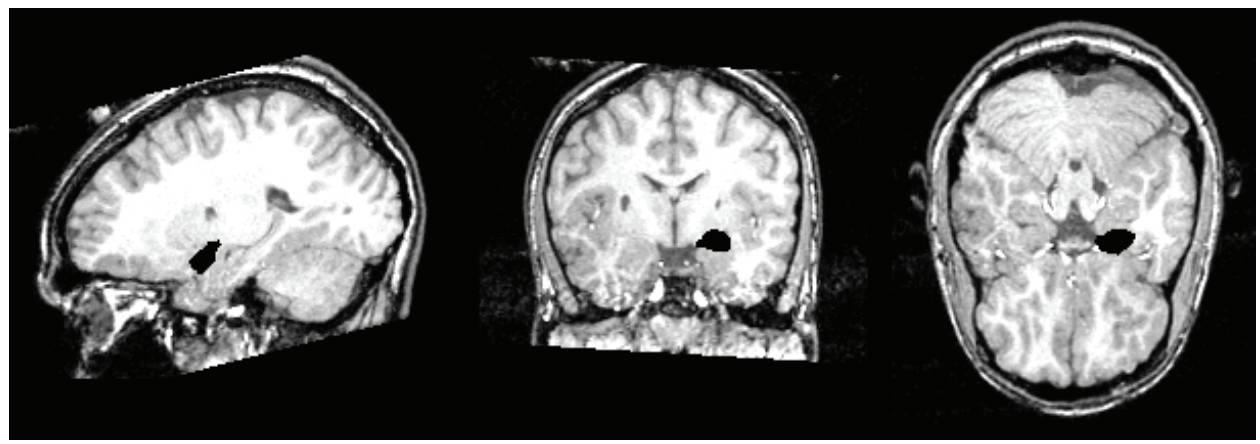

Figure 2: The manual segmentation of left amygdala at the mid-sagittal, coronal and axial cross-sections. The amygdala was segmented using a prior information of adjacent structures such as anterior commissure and hippocampus (Nacewicz et al., 2006).

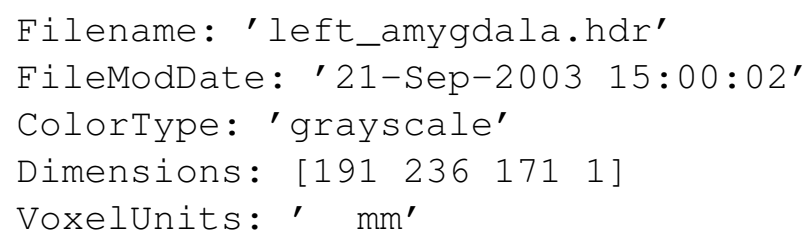

The structure array header contains various image information and can be accessed by calling, for instance, header.Dimensions which shows the dimension of array [ $191236 \quad 171$ l $]$. The last dimension is reserved for temporally changing 3D volume images such as fMRI but it is not used for this example. To extract the amygdala segmentation and saved into a 3D array vol of size $191 \times 236 \times 171$, we run

>>vol=analyze75read (header);

vol is then a 3D binary array of a left amygdala consisting of zeros and ones (Figure 2). The array vol consists of all zeros except the voxels where amygdala is defined.

\subsection{Amygdala Volume Data}

This is the subset of the 44 subject data set first published in Chung et al. (2010) consisting of the both left and right amygdala binary volume of 22 subjects. There are 22 normal control subjects and 24 autistic subjects. The data set is stored as amygdala. volume. data containing variables age, total brain volume (bra in), 
eye fixation duration (eye), face fixation duration ( $f a c e$ ), group variable indicating autistic or normal control (group), left amygdala volume (leftvol) and right amygdala volume (rightvol) data. The main scientific hypothesis of interest is to quantify volume and shape differences between the groups. In this section, we will show how to load the data set and do a simple volume computation. Since the image resolution is $1 \times 1 \times 1 \mathrm{~mm}$, the amygdala volume is simply computed by counting the number of voxels belonging to the amygdala.

load amygdala.volume.mat

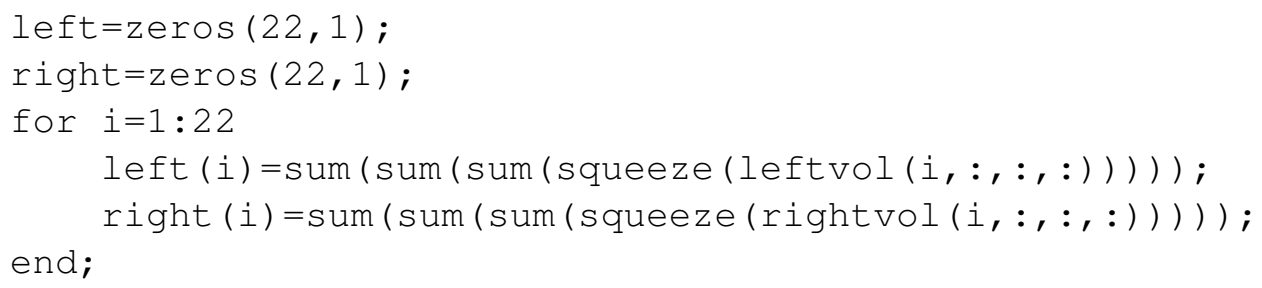

The group variable is 1 for autistic and 0 for normal controls. The left amygdala volume is computed and stored as al for instance. The amygdala volumes for all subjects are displayed in Figure 3 showing no clear separation of the groups.

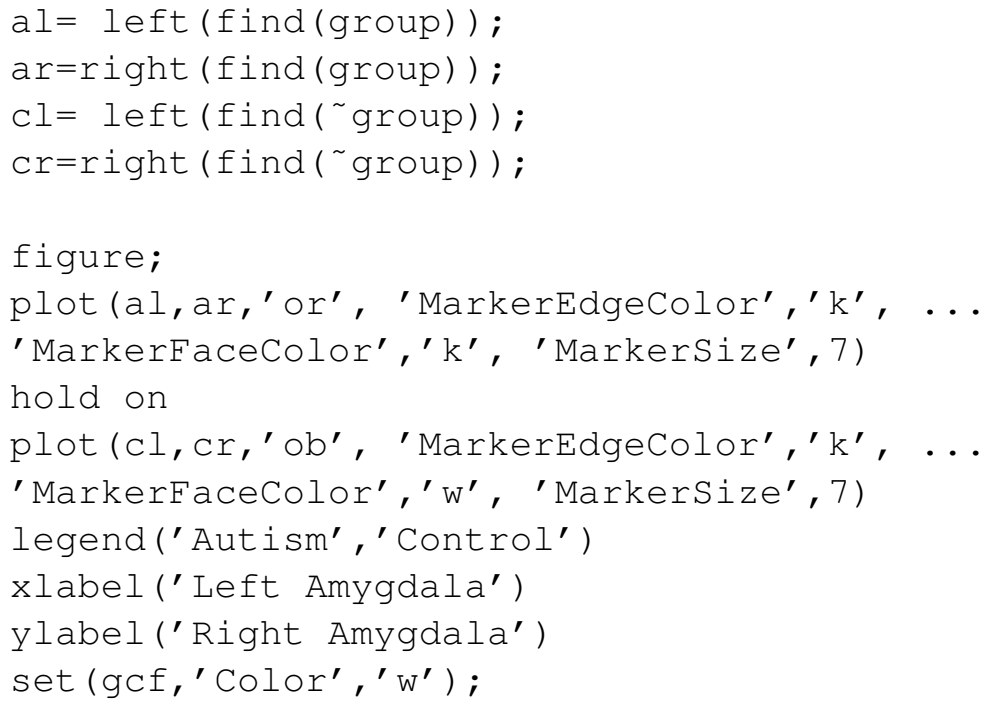

A more advanced technique such as weighted spherical harmonic representation (Chung et al., 2007, 2010) is needed to increase the discrimination power. 


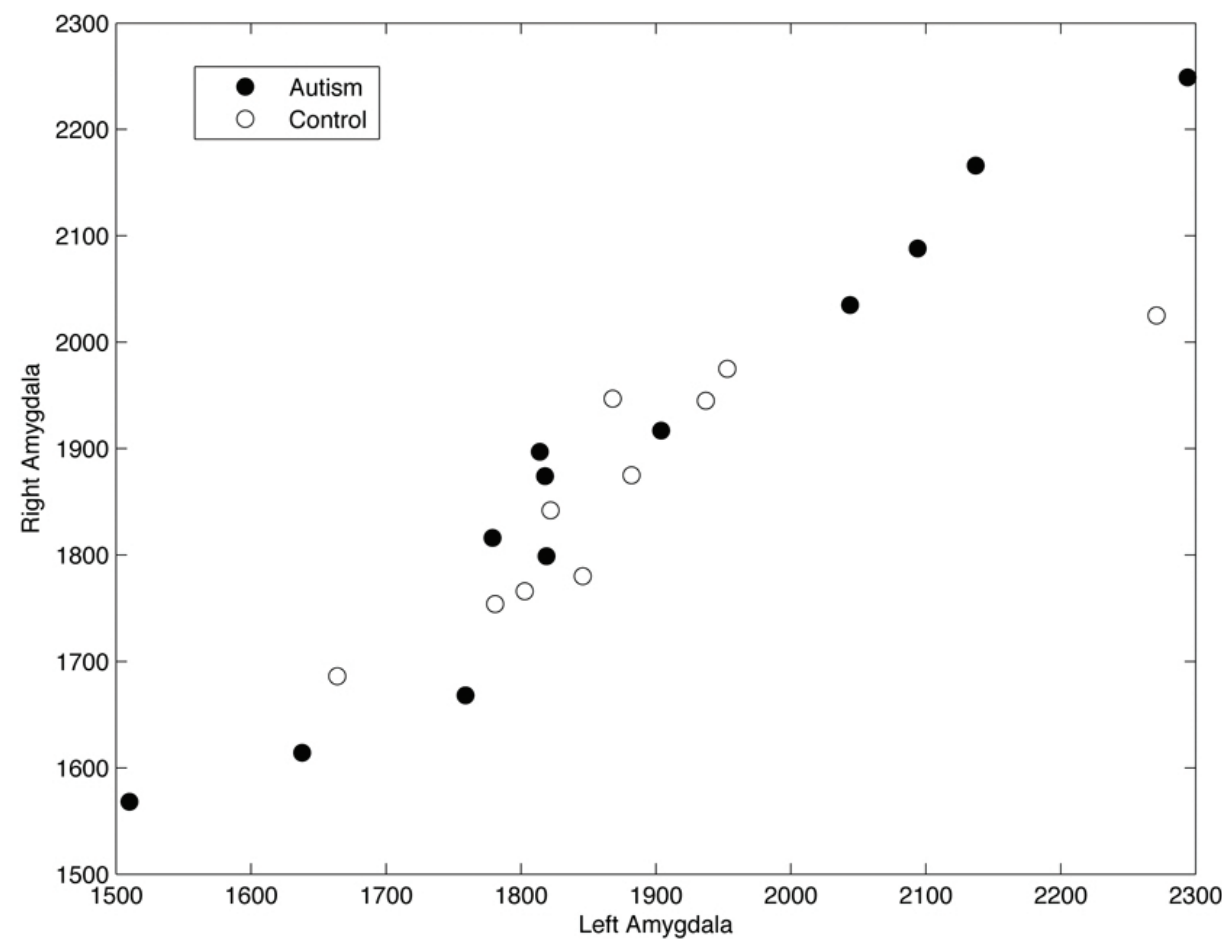

Figure 3: The plot showing the left and right amygdala volumes of autistic (black) and control (white) subjects. There is no visible group differences in the plot. The volume based method is not effective in discriminating between the groups.

\section{Surface Mesh Data}

Simple 3D neuroanatomical objects like hippocampus and amygdala can be represented by their $2 \mathrm{D}$ boundary as triangle meshes using the marching cubes algorithm (Lorensen and Cline, 1987), which is implemented in MATLAB.

To extract the surface mesh out of vol, we run

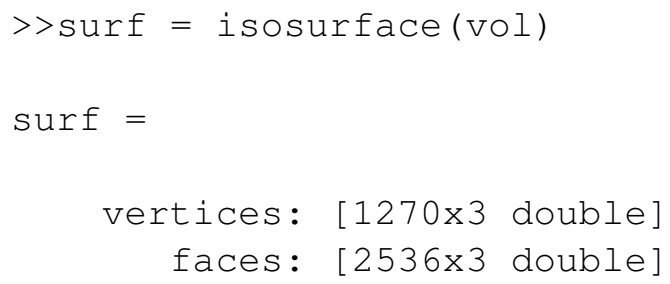


The MATLAB function isorsurface extracts the boundary of the amygdala and represents it as the structure array surf consisting of 1270 vertices and 2563 triangles. The visualization can be done with

>>figure_wire(surf,'yellow')

resulting in a surface mesh like Figure 4.

More complex neuroanatomical objects like cortical surfaces are difficult to represent with such a simple algorithm. Substantial research have been done on extracting cortical surfaces from MRI (Chung et al., 2003; Davatzikos and Bryan, 1995; MacDonald et al., 2000). The human cerebral cortex has the topology of a 2D highly convoluted grey matter shell with an average thickness of $3 \mathrm{~mm}$. The outer boundary of the shell is called the outer cortical surface while the inner boundary is called the inner cortical surface. The outer cortical surface is the boundary between the cerebrospinal fluid (CSF) and the gray matter while the inner cortical surface is the boundary between the gray and the white matters. Cortical surfaces are segmented from MRI using mainly a deformable surface algorithm and represented as a triangle mesh consisting of more than 40,000 vertices and 80,000 triangle elements (Chung et al., 2003; MacDonald et al., 2000). We assume cortical surfaces to be smooth 2D Riemannian manifolds topologically equivalent to a unit sphere (Davatzikos and Bryan, 1995). The triangle mesh format contains information about vertex indices, the Cartesian coordinates of the vertices and the connectivity that tells which three vertices form a triangle.

\subsection{Topology of Surface Data}

Surface extraction from MRI may cause possible topological defects such as handles and bridges forming on brain surfaces. So it is crucial to able to determine topological defects. Determining topological defects on the surface can be done by checking the topological invariants such as the Betti numbers or Euler characteristics.

Let $\chi$ be the Euler characteristic of a surface. The sphere has a Euler characteristic of 2. If the surface has genus $g$, the number of handles, the Euler characteristic is given by

$$
\chi=2-2 g .
$$

Each handle in the object reduces $\chi$ by 2 while the increase of the disconnected components raise $\chi$ by 2 . On the surface mesh, the Euler characteristic is given in terms of the number of vertices $V$, the number of edges $E$ and the number of faces $F$ using the polyhedral formula:

$$
\chi=V-E+F .
$$



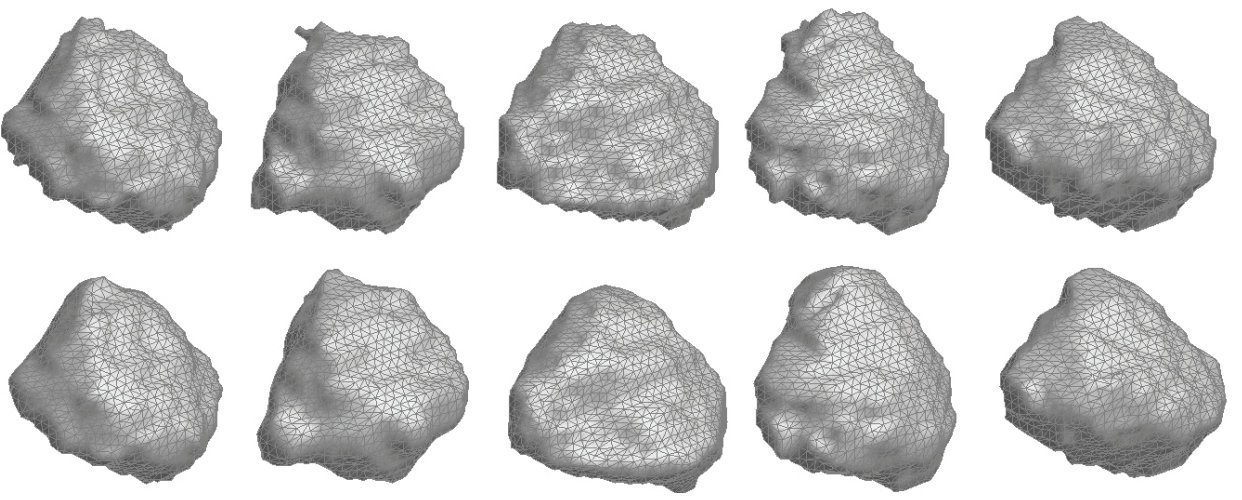

Figure 4: Top: triangle mesh representation of five different left amygdala surfaces. The mesh noises such as sharp peaks need to be smoothed out using surface smoothing technique. Here we used the weighted-SPHARM representation (Chung et al., 2010), which will be studied in a later chapter.

Note that for each triangle, there are three edges. For a closed surface topologically equivalent to a sphere, two adjacent triangles share the same edge. Hence, the total number of edges is $E=3 F / 2$. The relationship between the number of vertices and the triangles is $F=2 V-4$. We simply need to compute the Euler characteristic as $\chi=V-F / 2$ and check if it is 2 at the end. All binary volumes produced the topologically correct surfaces without an exception. Fig. 5 shows an example of before and after the topology correction.

For any type of cortical surface mesh, if $V$ is the number of vertices, $E$ is the number of edges, and $F$ is the number of faces or triangles in the mesh, the Euler characteristic $\chi$ of the mesh should be constant, i.e. $\chi=V-E+F=2$. Note that for each triangle, there are three edges. Since two adjacent triangles share the same edge, the total number of edges is $E=3 F / 2$. Hence, the relationship between the number of vertices and the triangles is $F=2 \mathrm{~V}-4$. In the sample surface, we have 40,962 vertices and 81,920 triangles.

The Euler characteristic computation in brain imaging has been traditionally done in connection with correcting for topological defects in segmented anatomical objects (Ségonne et al., 2007; Shattuck and Leahy, 2001). For instance, the human cerebral cortex has the topology of a 2D highly convoluted grey matter shell with an average thickness of $3 \mathrm{~mm}$. The outer and inner boundaries are assumed to be topologically equivalent to a sphere (Davatzikos and Bryan, 1995; MacDonald et al., 2000). Image acquisition and processing artifacts, and partial voluming will 

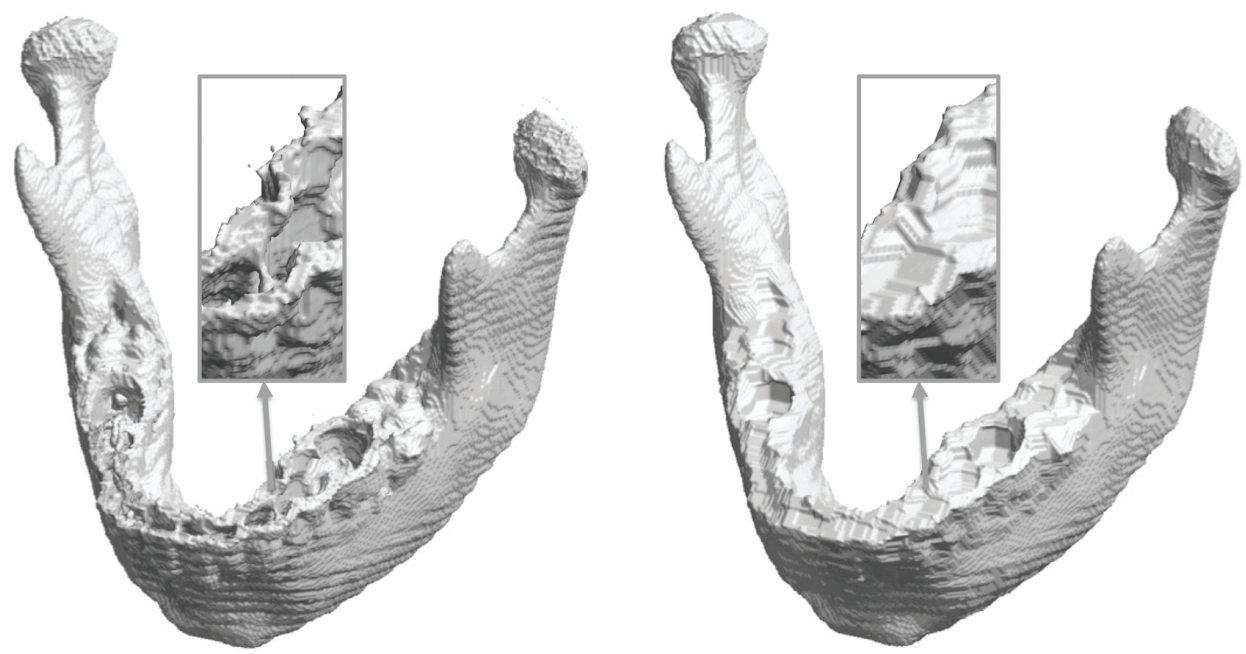

Figure 5: Topological defects occur in any type of medical image segmentation. Bridges and handles are visible in the teeth regions of the mandible segmentation obtained from CT images. Topological correction is made on mandible binary segmentation and surface. Disjointed tiny speckles of noisy components are removed by labeling the largest connected component, and holes and handles are removed by the morphological closing operation.

likely produce topological defects such as holes and handles in cortical segmentation. Since it is also needed to remove the brain stem and other parts of the brain in the triangulated mesh representation of the cortex, the resulting cortical surfaces are not likely to have spherical topology. So it is necessary to automatically determine and correct the topological defects using the topological invariants such as the Euler characteristic.

Topological defects are often encountered in image reconstruction. For instance, since the mandible and teeth have relatively low density in CT, unwanted cavities, holes and handles can be introduced in mandible segmentation (Andresen et al., 2000). An example is shown in Figure 5 where the tooth cavity forms a bridge over the mandible. In mandibles, these topological noises can appear in thin or cancellous bone, such as in the condylar head and posterior palate (Stratemann et al., 2010). If we apply the isosurface extraction on the topologically defect segmentation results, the resulting surface will have many tiny handles (Wood et al., 2004; Yotter et al., 2009). These handles complicate subsequent mesh operations such as smoothing and parameterization. So it is necessary to correct the 

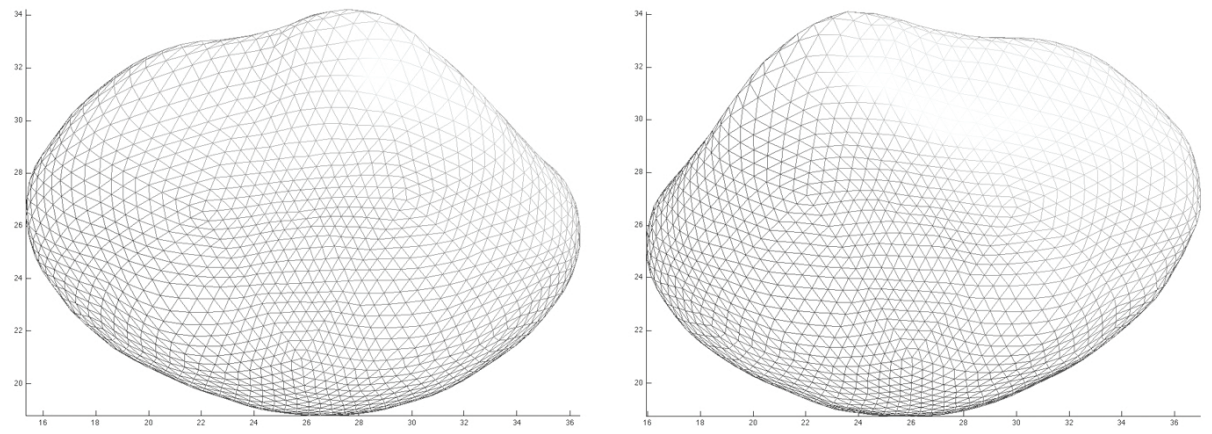

Figure 6: Left and right amygdala surface template constructed by averaging surface coordinates in the data set chung.2010.NI. mat.

topology by filling the holes and removing handles. If we correct such topological defects, it is expected the resulting isosurface is topologically equivalent to a sphere. There have been various topological correction techniques proposed in medial image processing. Rather than attempting to repair the topological defects of the already extracted surfaces (Wood et al., 2004; Yotter et al., 2009), we can perform the topological simplification on the volume representation directly using morphological operations (Guskov and Wood, 2001; Yotter et al., 2009). The direct correction on surface meshes can possibly cause surfaces to intersect with each other (Wood et al., 2004).

\subsection{Amygdala Surface Data}

This is the data set first published in (Chung et al., 2010) consisting of the both left and right amygdala surfaces of 44 subjects. There are 22 normal control subjects and 24 autistic subjects. All the amygdala surfaces went through image processing and meshes consist of 2562 vertices and 5120 faces. Mesh vertices anatomically match across subjects. So we can simply average the corresponding vertex coordinates to obtain the group average (Figure 6).

load chung.2010.NI.mat

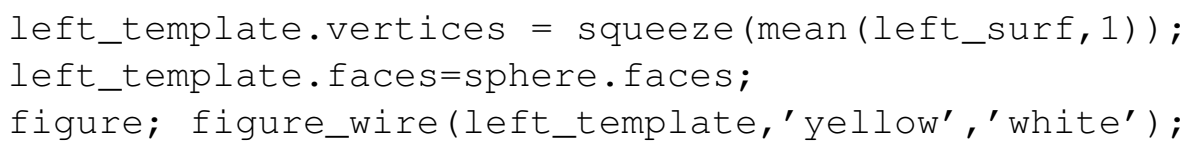



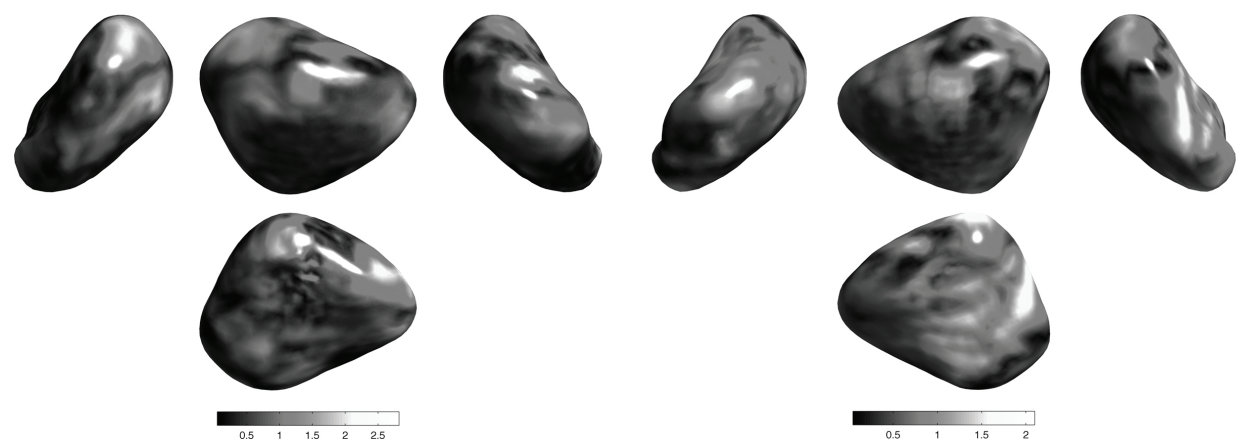

Figure 7: Displacement that is required to move the template surface to the first subject is displayed in the origami representation (Chung et al., 2010). The unit is in $\mathrm{mm}$ showing a fairly small deformation scale.

right_template.vertices = squeeze(mean(right_surf,1));

right_template.faces=sphere.faces;

figure; figure_wire(right_template, 'yellow', 'white') ;

The displacement vector field of warping the template surface to the 46 subject surfaces is given in $46 \times 2562 \times 3$ matrix. The displacement length of the first subject is shown in the origami representation introduced in (Chung et al., 2010) (Figure 7).

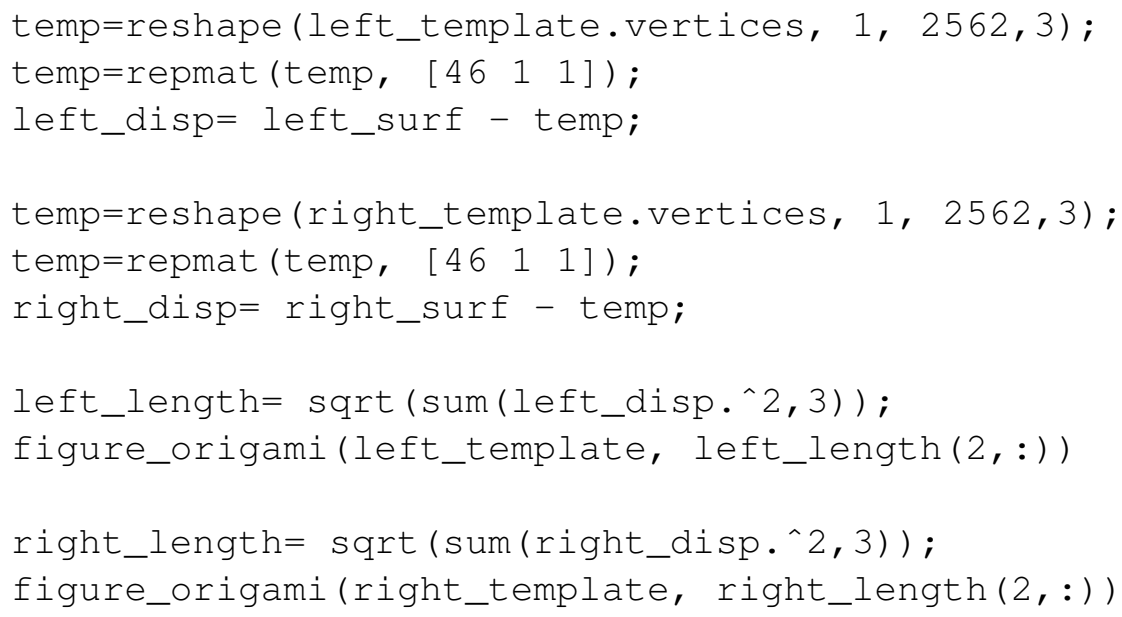




\section{Landmark Data}

Either manual or automatic landmarks that identify the important regions and features of anatomy are often used in landmark-based morphometrics. Landmarks are also used in aligning anatomy using affine registration. The landmark data introduced in this section is manually identified along the mandible surfaces extracted from CT images. The CT images were obtained from GE multi-slice helical CT scanners. CT scans were converted to DICOM format and subsequently Analyze 8.1 software package (AnalyzeDirect, Inc., Overland Park, KS) was used in segmenting binary mandible structure based on histogram thresholding. Then 24 landmarks are manually identified by an expert for two mandible surfaces. The landmarks across different surfaces anatomically correspond.

The mandible surfaces and the landmark data are stored in mandible-landmarks.mat. In the mat file, id contains the subject identifiers. id (44) is the subject F155-12-08 while id (1) is the subject F203-01-03. The mandible surfaces for these two subjects are shown in Figure 8. Our aim is to align them affinely as close as possible.

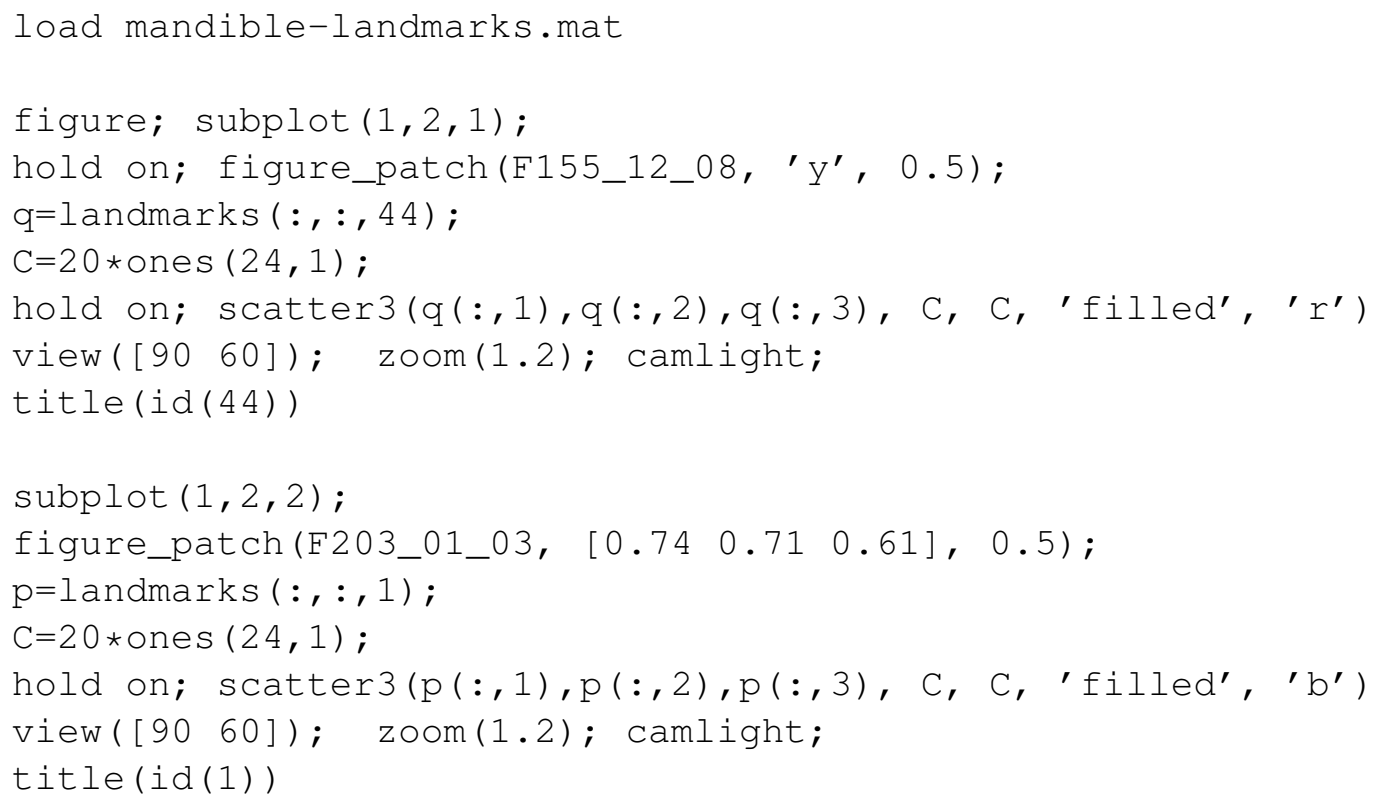

\subsection{Affine Transforms}

Anatomical objects extracted from 3D medical images are aligned using affine transformations to remove the global size differences. Figure 8 illustrates an ex- 
F155-12-08

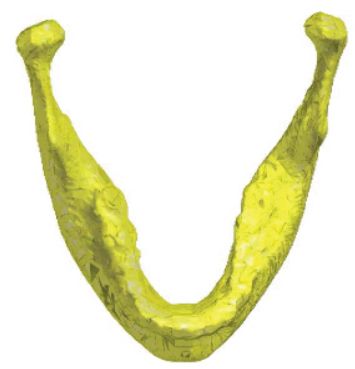

F203-01-03

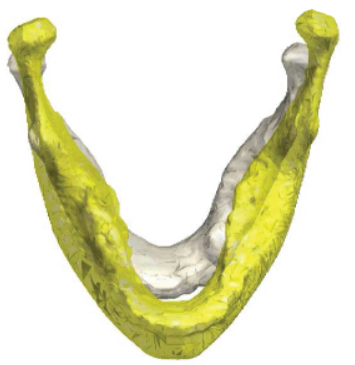

Affine transform

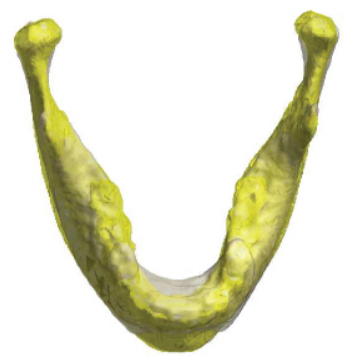

Figure 8: Mandible F155-12-08 (yellow) is used as a fixed template and other mandibles are affinely aligned to F155-12-08. For example, smaller F203-01-03 (gray) is aligned to larger F203-01-03 by affinely enlarging it.

F155-12-08

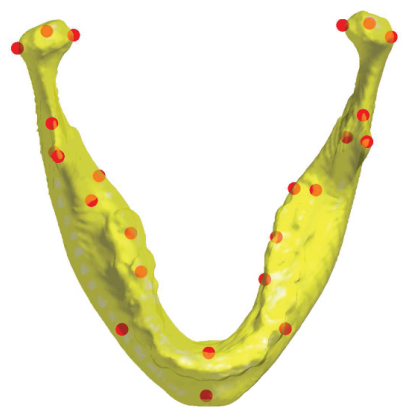

Affine transform of F203-01-03

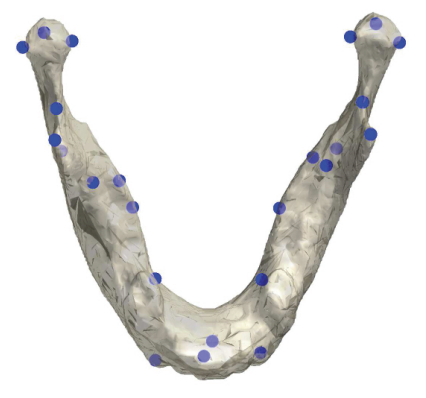

F203-01-03

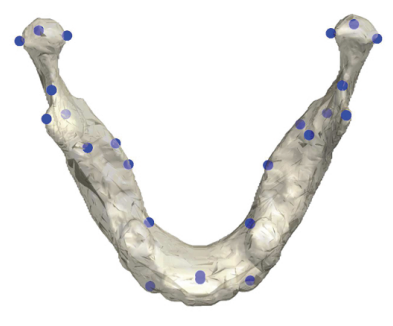

Landmark alignment

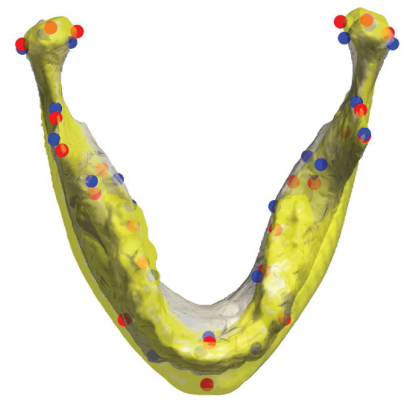

Figure 9: Mandible F155-12-08 (yellow) is used as a template and mandibles F20301-03 is affinely aligned to F155-12-08 by matching 24 manually identified landmarks. The affine transform does not exactly match the landmarks perfectly but minimizes the distance between them in a least squares fashion. 
ample where one mandible surface is aligned to another via an affine transform.

The affine transform $T$ of point $p=\left(p_{1}, \cdots, p_{d}\right)^{\prime} \in \mathbb{R}^{d}$ to $q=\left(q_{1}, \cdots, q_{d}\right)^{\prime}$ is given by

$$
q=R p+c,
$$

where the matrix $R$ corresponds to rotation, scaling and shear while $c$ corresponds to translation. Note that the affine transform is nonlinear. Note that

$$
\begin{aligned}
T(a p+b q) & =R(a p+b q)+c \\
& =a(R p+c)+b(R q+c)-c(a+b-1) \\
& =a T(p)+b T(q)-c(a+b-1) .
\end{aligned}
$$

Unless $a+b \neq 1$, the affine transform is not linear. However, the affine transform can be easily made into a linear form by augmenting the transform. The affine transform can be rewritten in a matrix form as

$$
\left(\begin{array}{c}
q \\
1
\end{array}\right)=\left(\begin{array}{cc}
R & c \\
0 \cdots 0 & 1
\end{array}\right)\left(\begin{array}{c}
p \\
1
\end{array}\right)
$$

The affine transformation matrix $A$ is then given by

$$
A=\left(\begin{array}{cc}
R & c \\
0 \cdots 0 & 1
\end{array}\right)
$$

Trivially $A$ is linear on $\left(\begin{array}{c}p \\ 1\end{array}\right)$. The matrix $A$ is the most often used form for affine registration in medical imaging. The inverse of the affine transform is given by

$$
p=R^{-1} q-R^{-1} c .
$$

This can be written in a matrix form as

$$
\left(\begin{array}{c}
p \\
1
\end{array}\right)=\left(\begin{array}{cc}
R^{-1} & -R^{-1} c \\
0 \cdots 0 & 1
\end{array}\right)\left(\begin{array}{c}
q \\
1
\end{array}\right) .
$$

We denote the matrix form of the inverse as

$$
A^{-}=\left(\begin{array}{cc}
R^{-1} & -R^{-1} c \\
0 \cdots 0 & 1
\end{array}\right) \text {. }
$$

Let $\mathbf{p}_{i}$ be the $i$-th landmark and its corresponding affine transformed points $\mathbf{q}_{i}$. Then we have

$$
\left(\begin{array}{c}
\mathbf{q}_{i} \\
1
\end{array}\right)=A\left(\begin{array}{c}
\mathbf{p}_{i} \\
1
\end{array}\right)
$$


Subsequently, we can estimate the affine transform matrix $A$ in the least squares fashion. The least squares estimation of $A$ is given by

$$
\widehat{A}=\arg \min _{A \in \mathcal{G}} \sum_{i=1}^{n}\left\|\left(\begin{array}{c}
\mathbf{q}_{i} \\
1
\end{array}\right)-A\left(\begin{array}{c}
\mathbf{p}_{i} \\
1
\end{array}\right)\right\|^{2},
$$

where $\mathcal{G}$ is an affine group. Since the direct optimization of this form is difficult, we alternately rewrite (4) as

$$
\underbrace{\left(\begin{array}{lll}
\mathbf{q}_{1} & \cdots & \mathbf{q}_{n}
\end{array}\right)}_{Q}=\left(\begin{array}{ll}
R & c
\end{array}\right) \underbrace{\left(\begin{array}{ccc}
\mathbf{p}_{1} & \cdots & \mathbf{p}_{n} \\
1 & \cdots & 1
\end{array}\right)}_{P} .
$$

Then the least squares estimation is trivially given as

$$
\left(\begin{array}{ll}
\widehat{R} & \widehat{c}
\end{array}\right)=Q P^{\prime}\left(P P^{\prime}\right)^{-1} \text {. }
$$

This can be easily implemented in MATLAB. Then the points $\mathbf{p}_{i}$ are mapped to $\widehat{R} \mathbf{p}_{i}+\widehat{c}$, which may not coincide with $\mathbf{q}_{i}$ in general.

If we try to solve the least squares problem with an additional constraint, the problem can become complicated. For instance, if we restrict $R$ to be rotation only, i.e. $R^{\prime} R=I$, iterative updates of least squares estimation are needed (Späth, 2004).

Affine transforms from the landmarks $p$ to $q$ is given computed as follows.

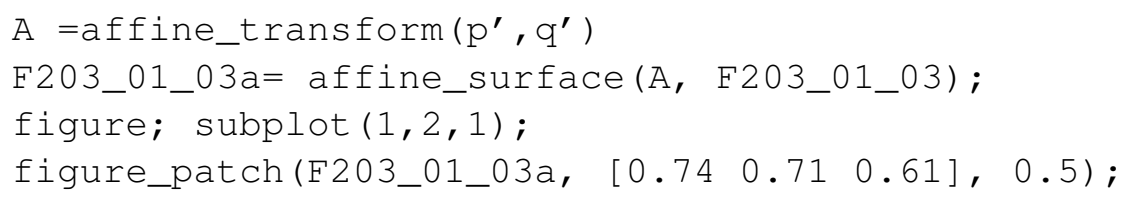

The estimated affine transform is then applied to the landmark coordinates $\mathrm{p}$. The transformed landmarks are named as paffine and displayed as follows.

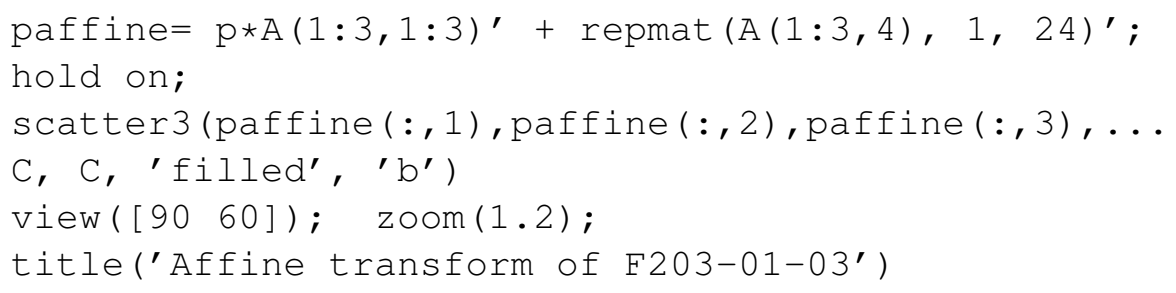

Let us superimpose the affine transformed landmarks and the mandible on top of F155-12-08. As we can see, the affine transformed landmarks paffine does not exactly match to $q$. However, the distance between $q$ and paffine is the smallest in the least squares fashion. 


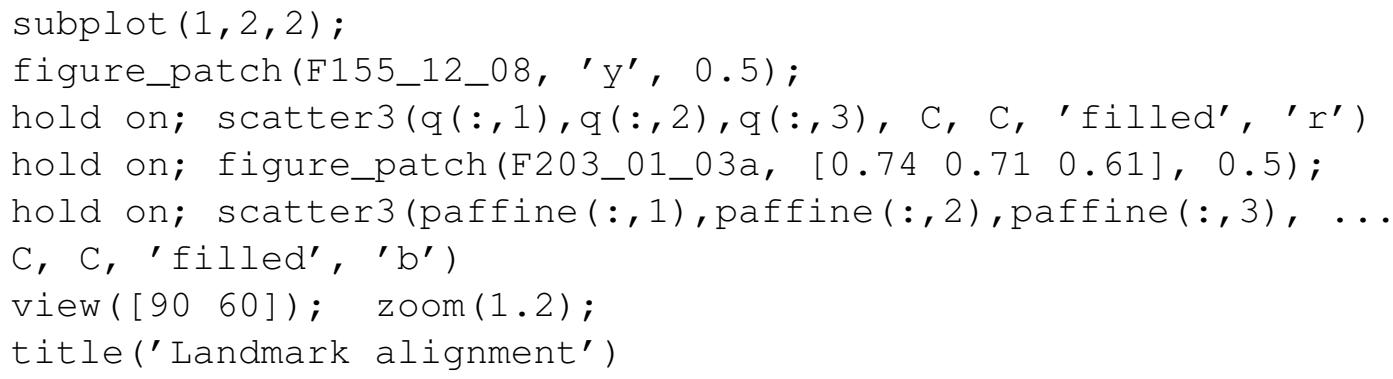

\section{Vector Data}

Vector image data are often obtained in connection with deformation-based morphometry (DBM) (Ashburner and Friston, 2000; Ashburner et al., 2000; Chung et al., 2001) which does not require tissue segmentation in quantifying the shape of anatomy in MRI (Ashburner and Friston, 2000). The 3D displacement vector field $u(x)$ is a vector map defined at each position $x$ that is required to move the anatomical structure at $x$ in an image $\mathcal{I}_{1}$ to the anatomically corresponding position in another image $\mathcal{I}_{2}$. The structure at $x$ in image $\mathcal{I}_{1}$ should match closely to the structure at $x+u$ in image $\mathcal{I}_{2}$. The displacement vector field $u$ is usually estimated via volume- or surface-based nonlinear registration techniques. The $x-, y$ and $z$-components of displacement vector fields are usually stored in three separate Analyze files. We will study DBM in a later chapter.

Here we briefly introduce the mandible surface deformation vector data set that will be used in the later chapter. mandible 77 sub ject. mat contains surface deformation vector data for 77 subjects obtained from CT images. The data set contains the vertex coordinates vertices, subject id id, voxel size voxelsize, mesh connectivity information faces and affine transform matrices A for all 77 subject. Since the surface meshes are constructed from CT images with varying image resolution, it is necessary to keep track of the image resolution information. The surfaces are already affinely registered so there are no global size differences. In order to extract and display the mandible surface of the first subject as in Figure 10 , we run

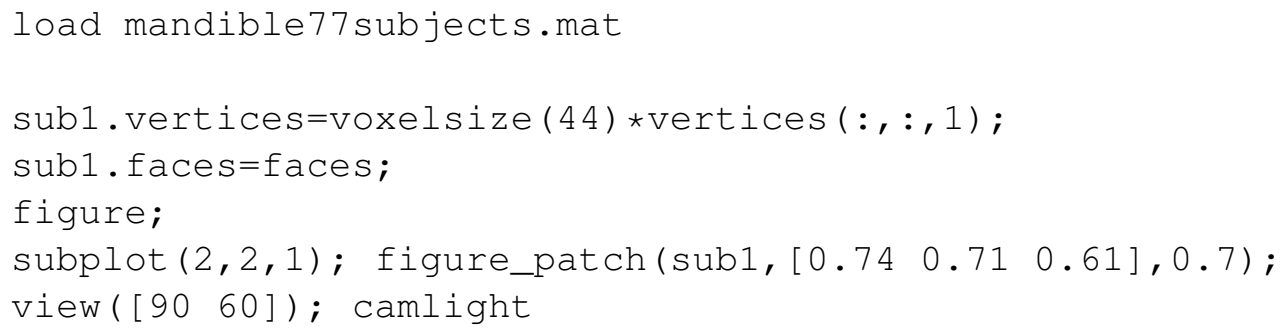



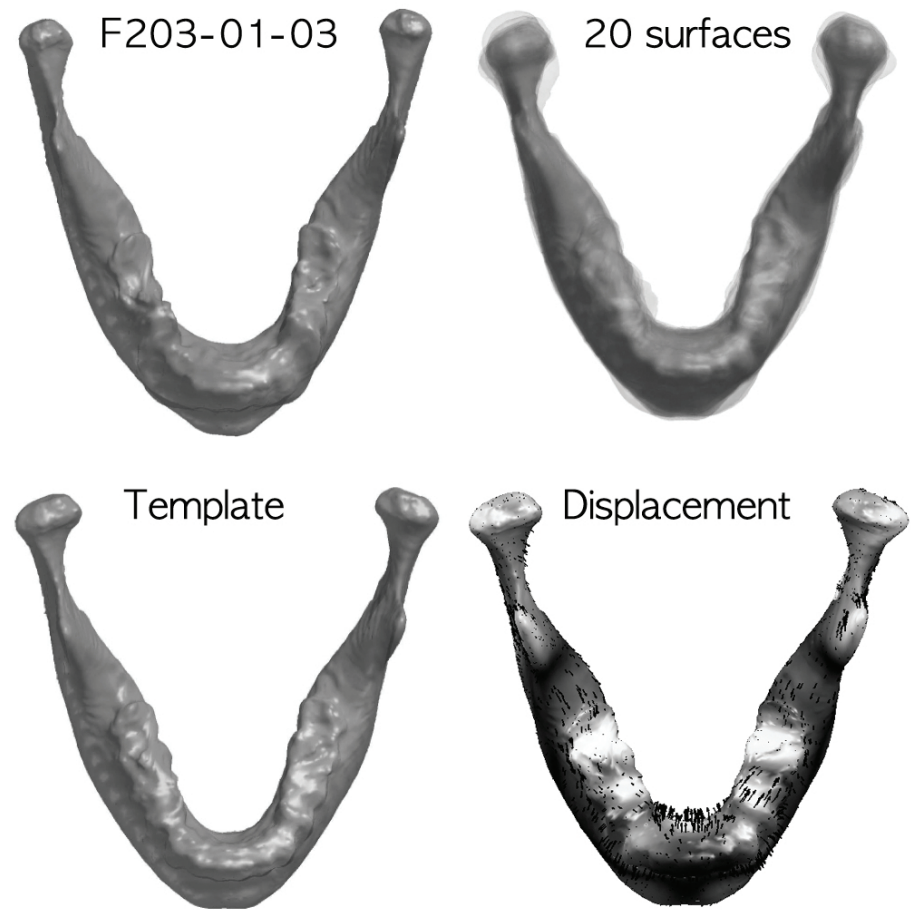

Figure 10: All 77 mandibles are affinely warped to F155-12-08 space. The affine transformed F203-01-03 surface is shown here. The superimposition of 20 affine registered mandibles shows local misalignment. Diffeomorphic registration is then performed to warp misaligned affine transformed mandibles. The surface meshes are then resampled in such a way that all the mandibles have identical mesh topology so that we can have anatomical correspondence across different mesh vertices. The average of deformation with respect to F155-12-08 provides the final population average template where statistical parametric maps will be constructed. The displacement vector from the template to F203-01-03 surface is shown as arrows.

title(id(1))

If we are interested in superimposing 20 surfaces (Figure 10), we run

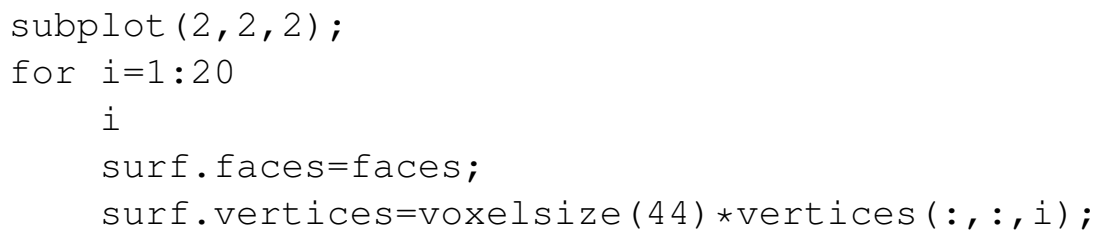




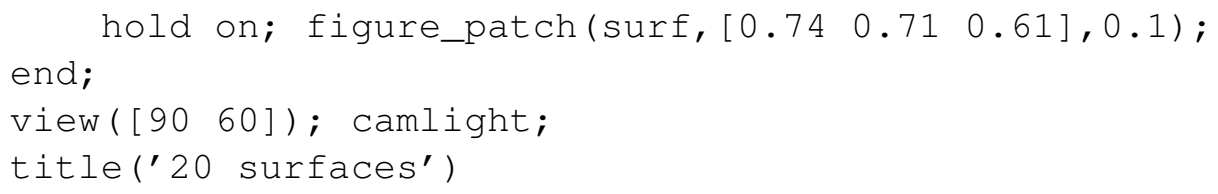

The template, which is the average of all 77 subjects, is given by averaging the surface coordinates in Figure 10:

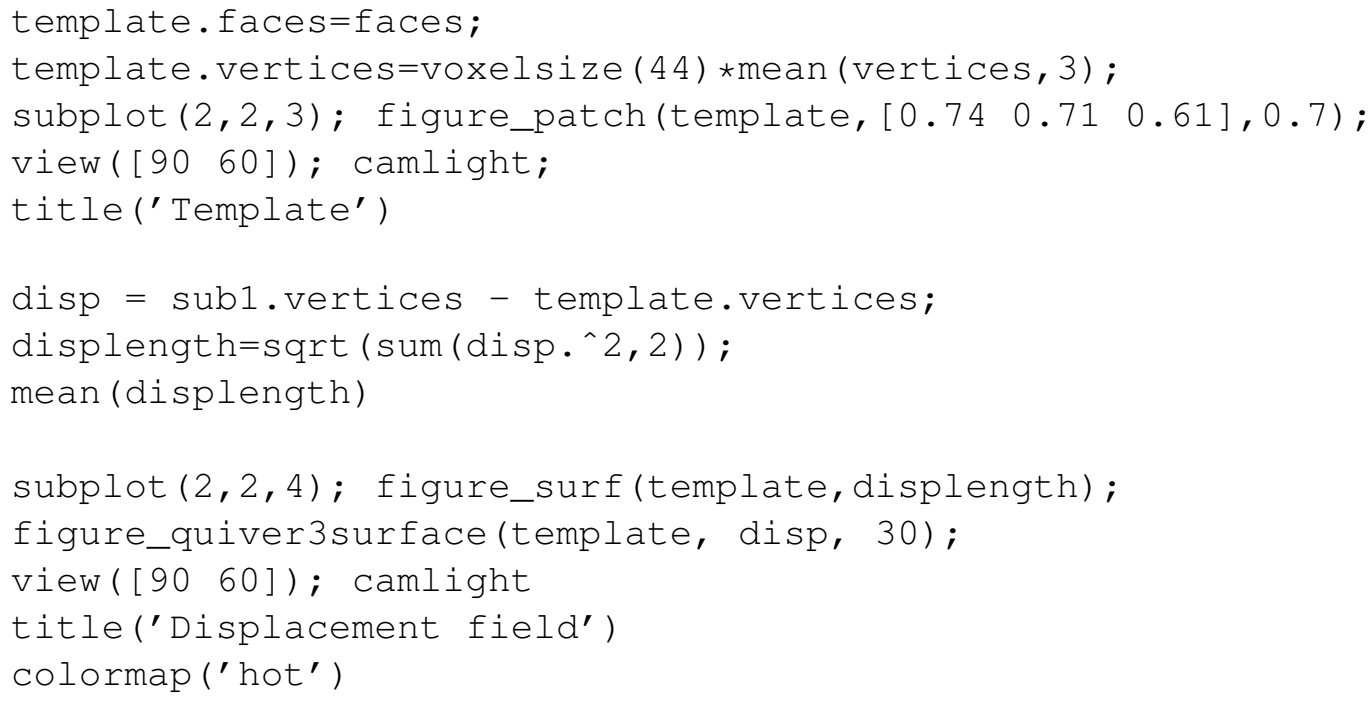

\section{Tensor and Curve Data}

Tensor and curve data are often obtained as the outcomes of diffusion tensor image (DTI) processing. DTI is a new imaging technique that has been used to characterize the macrostructure of biological tissues using magnitude, anisotropy and anisotropic orientation associated with water diffusion in the brain (Basser et al., 1994). DTI provides directional and connectivity information that MRI usually does not provide. The white matter fibers pose a physical constraint on the movement of water molecules along the direction of fibers. It is assumed that the direction of greatest diffusivity is most likely aligned to the local orientation of the white matter fibers (Mori and van Zijl, 2002). The directional information of water diffusion is usually represented as a symmetric positive definite $3 \times 3$ matrix $D=\left(d_{i j}\right)$ which is usually termed as the diffusion tensor or diffusion coefficients. The eigenvectors and eigenvalues of $D$ are obtained by solving

$$
D v=\lambda v,
$$


which results in 3 eigenvalues $\lambda_{1} \geq \lambda_{2} \geq \lambda_{3}$ and the corresponding eigenvectors $v_{1}, v_{2}, v_{3}$. The principal eigenvector $v_{1}$ determines the direction of the water diffusion, and is mainly used in streamline based tractography. Since there are exactly six unique elements in the diffusion tensor $D$, these six components of DTI are usually stored in six separate Analyze files.

From DTI, we can obtain the fractional anisotropy (FA) map, which is defined as

$$
\mathrm{FA}=\sqrt{\frac{\left(\lambda_{1}-\lambda_{2}\right)^{2}+\left(\lambda_{2}-\lambda_{3}\right)^{2}+\left(\lambda_{3}-\lambda_{1}\right)^{2}}{2\left(\lambda_{1}^{2}+\lambda_{2}^{2}+\lambda_{3}^{2}\right)}} .
$$

FA value is a scalar measure between 0 and 1 and it measures the amount of anisotropicity of diffusion.

We have provided a sample FA-map, which is used as a study template in (Chung et al., 2011). The FA-map is stored in NIfTI-1 file format and read using nii package written by Jimmy Shen of Rotman Research Institute (http: //research.baycrest.org/ jimmy/NIfTI). NIfTI-1 format is adapted from the widely used ANALYZE 7.5 file format (http://nifti.nimh.nih. gov).

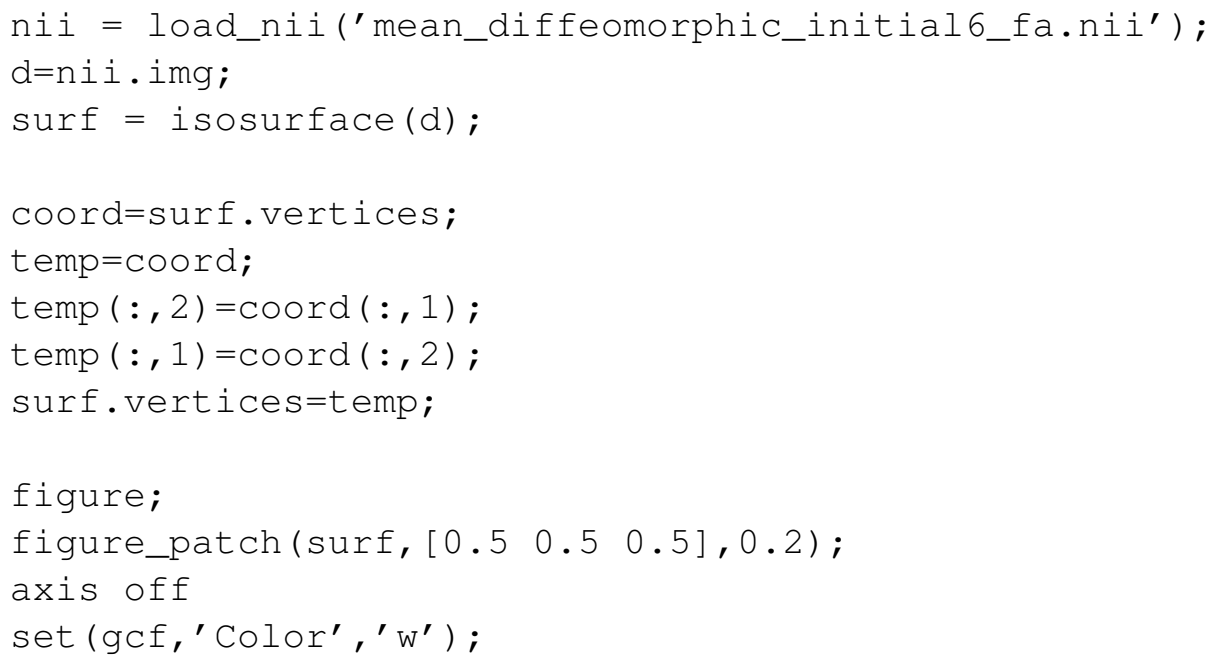

We have to swap $x$ - and $y$-coordinates of the image since the MATLAB convention is slightly different from the usual brain imaging format.

DTI is usually processed again to obtain the streamline based tractography (Lazar et al., 2003; Cook et al., 2006). Figure 11 shows the subsampled tractography result for a single subject. We will superimpose white matter fiber bundles stored in SL.mat on top of the FA-map. SL. mat contains 10000 white matter 

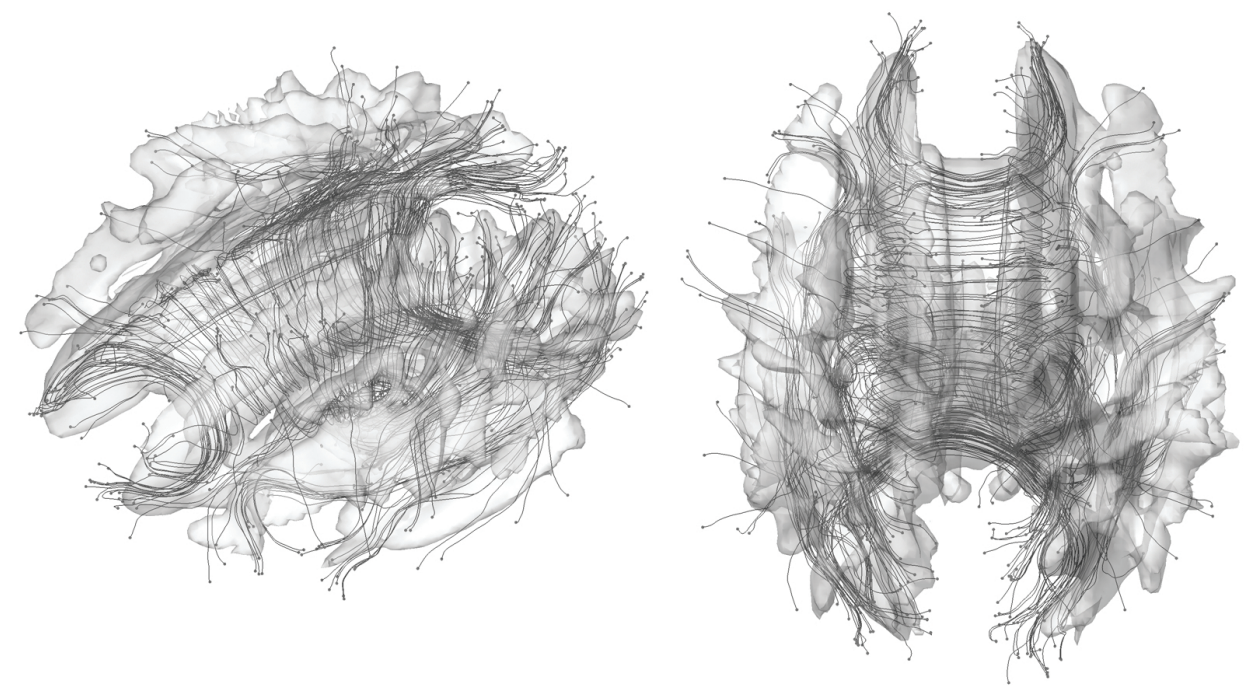

Figure 11: White matter fiber bundles obtained using TEND algorithm (Lazar et al., 2003; Cook et al., 2006). The end points of tracts are identified and colored as red. The surface is the isosurface of the template FA map so most of tracts are expected to end outside of the surface. The $\epsilon$-neighbor method uses the proximity of the end points in constructing the network graph.

fiber tracts stored as the cell data structure. Since there are too many tracts, we subsample for every 30 tracts and plot them.

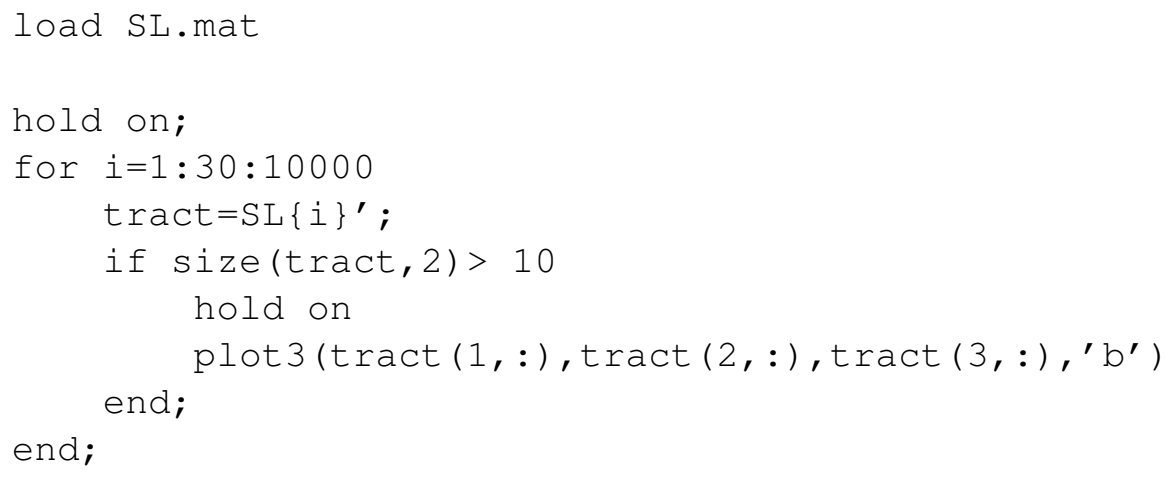




\section{Brain Image Analysis Tools}

Various neuroimage processing and analysis packages have been developed over the years. The main depository of neuroimaging tools is www.nitrc.org. The SPM (www.fil.ion.ucl.ac.uk/spm), AFNI (afni.nimh.nih.gov) and FSL (www. fmrib.ox.ac.uk/fsl) packages have been mainly designed for the whole brain volume based processing and univariate linear model type of analyses. The traditional statistical inference is then used to test hypotheses about the parameters of the model parameters. Although SPM and AFNI are probably the two most widely used analysis tools, their analysis pipelines are mainly based on a univariate general linear model and they do not have a routine for a multivariate or more complex statistical analysis. They also do not have the subsequent routine for correcting multiple comparison corrections for the multivariate linear models yet.

Unlike 3D whole brain volume based tools such as SPM, AFNI and FSL there are few cortical surface based tools such as the surface mapper (SUMA) (Saad et al., 2004) and FreeSurfer (surfer.nmr.mgh.harvard.edu). FreeSurfer is a widely used cortical surface segmentation and analysis tool. It consists of various image processing and segmentation tools. Also the spherical harmonic modeling tool SPHARM-PDM (www . ia . unc . edu/dev/download/shapeAnalys is) is available. However, these surface tools mainly do image processing and mesh representation and do not have the support for advanced statistical analyses.

For advanced multivariate linear modeling, for instance, one has to actually use statistical packages such as Splus (www . insight ful . com), R (www.r-project. org) and SAS (www. sas. com). These statistical packages do not interface with imaging data easily so the additional processing step is needed to read and write imaging data within the software. Further these tools do not have the random field based multiple comparison correction procedures so the users will likely export statistics maps to SPM or fMRISTAT (www.math.mcgill.ca/keith/ fmristat) increasing the burden of additional processing steps.

\subsection{Surfistat}

SurfStat package (www.stat.uchicago.edu/ worsley/surfstat) was developed by the late Keith J. Worsley of McGill University utilizing a model formula and avoids the explicit use of design matrices and contrasts, which tend to be a hinderance to most end users not familiar with such concepts. Probably this is the most sophisticated statistical tool for analyzing brain images. SurtStat can import MNI (MacDonald et al., 2000), FreeSurfer (surfer.nmr.mgh . harvard.edu) based cortical mesh formats as well as other volumetric image 
data. The model formula approach is implemented in many statistics packages such as Splus (www. insightful.com), R (www.r-project.org) and SAS (www.sas.com). These statistics packages accept a linear model like

$$
\mathrm{P}=\text { Group }+ \text { Age }+ \text { Brain }
$$

as the direct input for linear modeling avoiding the need to explicitly state the design matrix. $\mathrm{P}$ is a $n \times 3$ matrix of coordinates, Age is the age of subjects, Brain is the total brain volume of subject and Group is the categorical group variable $(0=$ control, $1=$ autism $)$. This type of model formula has yet to be implemented in widely used SPM or AFNI packages.

The other major novelty of the SurfStat package is the inclusion of mixedeffects models that can explicitly model the within-subject correlation of image scans of the same subject. SurfStat package is therefore better suited for longitudinally collected study designs than other packages.

\subsection{Public Image Database}

Other than the data sets we provided in the book, a large longitudinal image dataset is also available to the public. Open Access Series of Imaging Studies (OASIS, www.oasis-brains.org) and Alzheimer's Disease Neuroimaging Initiative (ADNI, www. loni.ucla.edu/ADNI) are two widely distributed data sets. The ADNI was initiated in 2003 by the National Institute on Aging (NIA) and the National Institute of Biomedical Imaging and Bioengineering (NIBIB) to determine if longitudinally collected MRI, PET and neuropsychological measurements can predict the progression of mild cognitive impairment (MCI) and Alzheimer's disease (AD) (www.adni-info.org). There is also a Simulated Brain Database (SBD) (brainweb.bic.mni.mcgill.ca/brainweb). The SBD contains a set of realistic MRI data volumes produced by an MRI simulator. These data can be used by the neuroimaging community to evaluate the performance of various image analysis methods in a setting where the truth is known.

\section{Acknowelgement}

This study was partially supported by NIH grants R01 EB022856 and R01 EB028753, and NSF grant MDS-2010778. 


\section{References}

P. R. Andresen, F. L. Bookstein, K. Conradsen, B. K. Ersbøll, J. L. Marsh, and S. Kreiborg. Surface-bounded growth modeling applied to human mandibles. IEEE Transactions on Medical Imaging, 19:1053-1063, 2000.

J. Ashburner and K. Friston. Voxel-based morphometry - the methods. NeuroImage, 11:805-821, 2000.

J. Ashburner, P. Neelin, D.L. Collins, A.C. Evans, and K.J. Friston. Incorporating prior knowledge into image registration. NeuroImage, 6:344-352, 1997.

J Ashburner, C. Good, and K.J. Friston. Tensor based morphometry. NeuroImage, 11S:465, 2000.

P.J. Basser, J. Mattiello, and D. LeBihan. MR diffusion tensor spectroscopy and imaging. Biophys J., 66:259-267, 1994.

M.A. Bernstein, K.E. King, X.J. Zhou, and W. Fong. Handbook of MRI pulse sequences. Medical Physics, 32:1452, 2005.

M.K. Chung. Statistical and Computational Methods in Brain Image Analysis. CRC Press, 2013.

M.K. Chung, K.J. Worsley, T. Paus, D.L. Cherif, C. Collins, J. Giedd, J.L. Rapoport, and A.C. Evans. A unified statistical approach to deformation-based morphometry. NeuroImage, 14:595-606, 2001.

M.K. Chung, K.J. Worsley, S. Robbins, T. Paus, J. Taylor, J.N. Giedd, J.L. Rapoport, and A.C. Evans. Deformation-based surface morphometry applied to gray matter deformation. NeuroImage, 18:198-213, 2003.

M.K. Chung, K.M. Dalton, A.L. Alexander, and R.J. Davidson. Less white matter concentration in autism: 2D voxel-based morphometry. NeuroImage, 23:242251, 2004.

M.K. Chung, K.M. Dalton, L. Shen, A.C. Evans, and R.J. Davidson. Weighted Fourier representation and its application to quantifying the amount of gray matter. IEEE Transactions on Medical Imaging, 26:566-581, 2007.

M.K. Chung, K.J. Worsley, M.N. Brendon, K.M. Dalton, and R.J. Davidson. General multivariate linear modeling of surface shapes using SurfStat. NeuroImage, 53:491-505, 2010. 
M.K. Chung, N. Adluru, K.M. Dalton, A.L. Alexander, and R.J. Davidson. Scalable brain network construction on white matter fibers. In Proc. of SPIE, volume 7962, page 79624G, 2011.

P.A. Cook, Y. Bai, S. Nedjati-Gilani, K.K. Seunarine, M.G. Hall, G.J. Parker, and D.C. Alexander. Camino: Open-source diffusion-MRI reconstruction and processing. In 14th Scientific Meeting of the International Society for Magnetic Resonance in Medicine, 2006.

C. Davatzikos and R.N. Bryan. Using a deformable surface model to obtain a shape representation of the cortex. Proceedings of the IEEE International Conference on Computer Vision, 9:2122-2127, 1995.

C.D. Good, I.S. Johnsrude, J. Ashburner, R.N.A. Henson, K.J. Friston, and R.S.J. Frackowiak. A voxel-based morphometric study of ageing in 465 normal adult human brains. NeuroImage, 14:21-36, 2001.

I. Guskov and Z.J. Wood. Topological noise removal. In Graphics Interface, pages 19-26, 2001.

S.C. Johnson, L.C. Baxter, L. Susskind-Wilder, D.J. Connor, M.N. Sabbagh, and R.J. Caselli. Hippocampal adaptation to face repetition in healthy elderly and mild cognitive impairment. Neuropsychologia, 42:980-989, 2004.

K. Kollakian. Performance analysis of automatic techniques for tissue classification in magnetic resonance images of the human brain. Technical Report Master's thesis, Concordia University, Montreal, Quebec, Canada, 1996.

M. Lazar, D.M. Weinstein, J.S. Tsuruda, K.M. Hasan, K. Arfanakis, M.E. Meyerand, B. Badie, H. Rowley, V. Haughton, A. Field, B. Witwer, and A.L. Alexander. White matter tractography using tensor deflection. Human Brain Mapping, 18:306-321, 2003.

W.E. Lorensen and H.E. Cline. Marching cubes: A high resolution 3D surface construction algorithm. In Proceedings of the 14th Annual Conference on Computer Graphics and Interactive Techniques, pages 163-169, 1987.

J.D. MacDonald, N. Kabani, D. Avis, and A.C. Evans. Automated 3-D extraction of inner and outer surfaces of cerebral cortex from MRI. NeuroImage, 12:340$356,2000$.

A.B. McMillan, B.P. Hermann, S.C. Johnson, R.R. Hansen, M. Seidenberg, and M.E. Meyerand. Voxel-based morphometry of unilateral temporal lobe epilepsy reveals abnormalities in cerebral white matter. NeuroImage, 23:167-174, 2004. 
S. Mori and P.C.M. van Zijl. Fiber tracking: principles and strategies-a technical review. NMR in Biomedicine, 15:468-480, 2002.

B.M. Nacewicz, K.M. Dalton, T. Johnstone, M.T. Long, E.M. McAuliff, T.R. Oakes, A.L Alexander, and R.J. Davidson. Amygdala volume and nonverbal social impairment in adolescent and adult males with autism. Arch. Gen. Psychiatry, 63:1417-1428, 2006.

T. Paus, A. Zijdenbos, K.J. Worsley, D.L. Collins, J. Blumenthal, J.N. Giedd, J.L. Rapoport, and A.C. Evans. Structural maturation of neural pathways in children and adolescents: In vivo study. Science, 283:1908-1911, 1999.

D.A. Pizzagalli, T.R. Oakes, A.S. Fox, M.K. Chung, C.L. Larson, H.C. Abercrombie, S.M. Schaefer, R.M. Benca, and R.J. Davidson. Functional but not structural subgenual prefrontal cortex abnormalities in melancholia. Molecular Psychiatry, 9:393-405, 2004.

Z.S. Saad, R.C. Reynolds, B. Argall, S. Japee, and R.W. Cox. Suma: an interface for surface-based intra-and inter-subject analysis with afni. In IEEE International Symposium on Biomedical Imaging (ISBI), pages 1510-1513, 2004.

F. Ségonne, J. Pacheco, and B. Fischl. Geometrically accurate topology-correction of cortical surfaces using nonseparating loops. IEEE Transactions on Medical Imaging, 26:518-529, 2007.

D.W. Shattuck and R.M. Leahy. Automated graph-based analysis and correction of cortical volume topology. IEEE Transactions on Medical Imaging, 20:1167$1177,2001$.

H. Späth. Fitting affine and orthogonal transformations between two sets of points. Mathematical Communications, 9:27-34, 2004.

S. A. Stratemann, J. C. Huang, K. Maki, D. C. Hatcher, and A. J. Miller. Evaluating the mandible with cone-beam computed tomography. American Journal of Orthodontics and Dentofacial Orthopedics, 137:S58-S70, 2010.

Z. Wood, H. Hoppe, M. Desbrun, and P. Schröder. Removing excess topology from isosurfaces. ACM Transactions on Graphics (TOG), 23:190-208, 2004.

R. A. Yotter, R. Dahnke, and C. Gaser. Topological correction of brain surface meshes using spherical harmonics. In Medical Image Computing and ComputerAssisted Intervention (MICCAI), volume 5762 of Lecture Notes in Computer Science, pages 125-132. Springer, 2009. 